\title{
Diagnosis and treatment of Wernicke's encephalopathy in an in-patient alcohol detoxification unit: a completed audit cycle
}

\author{
Vijay Delaffon, ${ }^{1}$ Srinivas Naik, ${ }^{1}$ Rajandeep Mann, ${ }^{1}$ Arshya Vahabzadeh, ${ }^{2}$ \\ Timothy Millward, ${ }^{1}$ Adeel Majeed, ${ }^{1}$ Angela Taylor, ${ }^{1}$ Anshuman Pant ${ }^{1}$
}

The Psychiatrist (2013), 37, 384-388, doi: 10.1192/pb.bp.111.035907

${ }^{1}$ Leicestershire Partnership NHS Trust, Leicester, UK; ${ }^{2}$ Emory University, Atlanta, USA

Correspondence to Vijay Delaffon (Vijay.Delaffon@kmpt.nhs.uk)

First received 5 Jul 2011, final revision 11 Mar 2013, accepted 24 Apr 2013

\begin{abstract}
Aims and method A baseline audit of trainees' ability to recognise Wernicke's encephalopathy and initiate appropriate thiamine regimes in an in-patient alcohol detoxification unit was carried out. Based on the findings, gaps were addressed using targeted education and training, and their impact on improving standards of managing Wernicke's encephalopathy was re-audited.
\end{abstract}

Results The initial audit revealed that trainees' ability to recognise Wernicke's encephalopathy and initiate thiamine was inadequate. Significant improvement in appropriate use of treatment regime $(P<0.05)$ of thiamine and the monitoring of clinical response was observed after addressing the initial gaps through education.

Clinical implications Treatment of Wernicke's encephalopathy can be inadequate, given a lack of awareness of clinical presentation and appropriate management. This can be addressed through education and training.

Declaration of interest None.
Wernicke's encephalopathy is an acute neuropsychiatric condition commonly associated with chronic alcohol misuse. Described in 1881 by Carl Wernicke, the syndrome is characterised by disturbances of eye movement and gait, polyneuropathy and mental changes which include confusion, apathy and decreased attention span. Failure to treat the condition adequately with thiamine can lead to Korsakoff syndrome in up to $84 \%$ of patients. ${ }^{1}$ This assumes significance in light of Cook et al's ${ }^{2}$ report that $25 \%$ of patients with Korsakoff syndrome require long-term institutionalised care. Mortality rates of up to $17 \%$ have been reported in Wernicke's encephalopathy. ${ }^{1}$

Thiamine deficiency as a causative factor was suggested by Campbell and Russell in 1940. The use of thiamine to treat Wernicke's encephalopathy is based on resolution of symptoms, as evidenced by published case reports. ${ }^{1,2}$ However, there is a lack of robust evidence to guide clinicians on the dose, frequency, route of administration and length of treatment regimes. Current practice therefore is based on extrapolations of basic science, case reports and treatment guidelines. ${ }^{3}$

The prevalence of Wernicke-Korsakoff syndrome is estimated to be $1-2 \%$ in the general population and may be higher, between 12.5 and $35 \%$ in people with alcohol dependence. ${ }^{1,2,4,5}$ Indirect evidence is available to indicate that Wernicke's encephalopathy is underrecognised and, consequently, undertreated. Studies point to an ante mortem diagnosis of Wernicke's encephalopathy in only $5-14 \%$ of patients. $^{6,7}$

The classic triad of confusion, ophthalmoplegia and ataxia is found in only a small proportion of patients with Wernicke's encephalopathy. From a survey of 427 physicians, Hope et $a l^{8}$ report that $21 \%$ of psychiatrists and $10 \%$ accident and emergency (A\&E) specialists required all three symptoms to be present in order to administer parenteral thiamine. This could contribute to the underrecognition and treatment of Wernicke's encephalopathy as only $10 \%$ of patients present with the full triad of symptoms. ${ }^{9}$ Among those with Wernicke's encephalopathy, $23 \%$ present with ataxia and $29 \%$ with eye signs. Kopelman et $a l^{10}$ highlight the issue of missed opportunities to intervene at an early stage in preventing long-term impairment in this population. These opportunities present in primary care, community alcohol services and also repeated presentations to $\mathrm{A} \& \mathrm{E}$ departments or hospital wards. Although neuroimaging and alcohol biomarkers have shown the potential in providing objective tools to inform and support diagnosis, there is a lack of application in clinical practice. ${ }^{11,12}$ Therefore, a high level of suspicion is required to diagnose Wernicke's encephalopathy when any of the symptoms are noticed with a history of alcohol misuse; this is in keeping with the Royal College of Physicians' guidelines for managing Wernicke's encephalopathy. ${ }^{13}$ 
Measures to improve current practice in treatment have been disappointing. Day et $a l^{14}$ report inadequate treatment of Wernicke's encephalopathy even with national guidelines and prompting by a pharmacist. They recommend regular education for all junior doctors and experienced clinicians in the assessment and management of the condition.

\section{Aims}

We set out to assess clinical practice in recognition of Wernicke's encephalopathy and initiation of thiamine treatment among trainees in the East Midlands Deanery (South). We then aimed to evaluate the impact of an educational intervention in improving recognition of Wernicke's encephalopathy and practice of thiamine prescribing among these trainees using an audit.

\section{Method}

The audit was carried out in the alcohol detoxification ward of the Bradgate Mental Health Unit, Glenfield Hospital, Leicester. We focused on this group, as the rate of Wernicke's encephalopathy is particularly high among those with greater severity of alcohol dependence and potential interventions would have the highest impact in that group. The audit proposal was approved by the Clinical Audit Group of Leicestershire Partnership NHS Trust.

Patients are admitted for a planned in-patient detoxification for a period of 7-10 days. We carried out a retrospective case-note audit of all patients admitted between January 2010 and March $2010(n=40)$ based on convenience sampling. Case notes were reviewed and data extracted regarding the following variables: age, gender, duration of current episode of alcohol dependence, average units of alcohol consumption per day and the presence of psychiatric and medical comorbidities. In addition, case notes were reviewed for documentation of signs and symptoms on initial admission and prescribed thiamine regimes were also recorded.

\section{Audit standards}

The diagnostic and prescribing practices were compared with the Trust's detoxification protocol which closely follows the Maudsley prescribing guidelines. ${ }^{15}$ Prophylactic regime was defined as one pair of high-potency B-complex vitamins intramuscularly for 3-5 days. All patients undergoing alcohol detoxification should have received this as a minimum.

Presence of one or more of the following symptoms: ataxia, confusion, ophthalmoplegia or nystagmus, memory disturbance, hypothermia or hypotension, coma or unconsciousness is sufficient to form a presumptive diagnosis of Wernicke's encephalopathy. ${ }^{15}$

Therapeutic regime of thiamine should be initiated for all patients with a presumptive diagnosis. This is defined as two pairs of high-potency B-complex vitamins intramuscularly for 2 days. If there was no evidence of response, treatment should have been discontinued. If any clinical response was noted, treatment should have been continued with one pair daily for 5 days or for as long as the improvement continued. Therefore, we also looked for documented evidence of ongoing monitoring of clinical signs in those fulfilling criteria for Wernicke's encephalopathy. Absence of documentary evidence was recorded as a negative for all variables.

The patients had been clerked by a typical range of junior doctors working within an adult in-patient psychiatric unit. Occasionally this would have been the team's own foundation year 2 or core trainee, but at other times this could have been the duty doctor who may have been a general practitioner (GP) trainee or another foundation year 2 doctor.

The results of the baseline audit prompted us to design a lecture-based session to be delivered for all trainees at induction. The 1-hour module was developed and delivered by a consultant psychiatrist and a higher trainee in addiction psychiatry at induction training held at 4-month intervals for the new intake of foundation trainees and 6-month intervals for GP and core psychiatry trainees. The aim was to improve the awareness of trainees regarding Wernicke's encephalopathy. Emphasis was placed on history-taking and focused examination, which in turn would help the trainee to identify Wernicke's encephalopathy and initiate the appropriate treatment regime. In addition, recognition and management of Wernicke's encephalopathy was highlighted at weekly supervision which junior trainees based on the in-patient detoxification unit receive routinely. A re-audit was carried out on all patients admitted between May and July $2010(n=55)$ and the results were compared with the baseline audit.

The re-audit used data from admission notes documented by a different set of junior doctors to the first audit. These trainees would have received the lecture and the weekly supervision. It is acknowledged that this is a limitation, and is explored further in the discussion section of this paper. Nontheless, this was unavoidable due to the rotation of junior doctors and as they were of a similar mix training stages we felt that their attitudes and skills would be comparable.

\section{Results}

The main demographic characteristics of the pre- and posttraining samples (I and II respectively) are highlighted in Table 1. The two samples do not differ significantly except in the average units of alcohol consumed per day. Nearly half of both samples had psychiatric comorbidities, with depression (40\% sample I and $27 \%$ sample II) and anxiety (20\% sample I and $27 \%$ sample II) the most common diagnoses. Unsurprisingly, alcoholic liver disease was the most common physical health comorbidity ( $17 \%$ sample I and $14 \%$ sample II).

The overall prevalence of presumptive Wernicke's encephalopathy in the audit population across both periods was $40 \% \quad(n=95)$ (Table 2). Eye signs were the most common clinical sign (32\%), whereas the classic triad was present in only 1 patient among 95 consecutive admissions over this 6-month period. Eye signs and ataxia were most commonly observed signs in combination $(32 \%, n=13)$.

In the pre-training period, $33 \%(n=13)$ of patients fulfilled clinical criteria for a presumptive diagnosis of Wernicke's encephalopathy on the basis of documentation in case notes as compared with $45 \%(n=25)$ in the posttraining period, although this was not statistically significant. 


\begin{tabular}{|c|c|c|}
\hline \multicolumn{3}{|c|}{$\begin{array}{l}\text { Demographic characteristics for the two audit } \\
\text { periods, pre- and post-training }\end{array}$} \\
\hline & $\begin{array}{l}\text { Pre-training, } \\
\qquad N=40\end{array}$ & $\begin{array}{l}\text { Post-training, } \\
\quad N=55\end{array}$ \\
\hline Age, years: mean (s.d.) & $40.9(11.6)$ & $41.1(8.0)$ \\
\hline $\begin{array}{c}\text { Gender, } n \\
\text { Male } \\
\text { Female }\end{array}$ & $\begin{array}{l}27 \\
13\end{array}$ & $\begin{array}{l}38 \\
17\end{array}$ \\
\hline $\begin{array}{l}\text { Duration of drinking } \\
\text { episode, months: mean (s.d.) }\end{array}$ & 82.5 (77.3) & $92.8(75.3)$ \\
\hline Alcohol units per day, mean (s.d.) & $33.3(16.3)$ & $42.4(22.5)$ \\
\hline
\end{tabular}

\begin{tabular}{|c|c|c|c|}
\hline \multirow[t]{2}{*}{ Table 2} & \multicolumn{3}{|c|}{$\begin{array}{l}\text { Clinical signs indicating presumptive Wernicke's } \\
\text { encephalopathy at initial assessment }\end{array}$} \\
\hline & & $\begin{array}{c}\text { Pre-training, } \\
N=40 \\
n(\%)\end{array}$ & $\begin{array}{c}\text { Post-training, } \\
\begin{array}{c}N=55 \\
n(\%)\end{array}\end{array}$ \\
\hline \multicolumn{2}{|c|}{ Acute confusion } & $0(0)$ & $3(6)$ \\
\hline \multicolumn{2}{|c|}{ Ocular abnormalities } & $13(3)$ & $18(33)$ \\
\hline \multicolumn{2}{|l|}{ Ataxia } & $10(25)$ & $18(33)$ \\
\hline \multicolumn{2}{|c|}{$\begin{array}{l}\text { Presumptive diagnosis } \\
\text { of Wernicke's encephalopathy }\end{array}$} & $13(33)$ & $25(45)$ \\
\hline
\end{tabular}

Among these, 6 patients (47\%) were appropriately treated in the pre-training period, i.e. with therapeutic doses of thiamine (Table 3). This number went up to $21(84 \%)$ post-training. In the pre-training period, $78 \%(n=21)$ of patients received appropriate prophylactic doses, whereas post-training this rose to $97 \%(n=29)$.

Observant readers will note that $68 \%(n=27)$ of patients in the pre-training period and 90\% $(n=50)$ in the post-training period received either regime. In the pre-training group, four patients with Wernicke's encephalopathy refused treatment regimes and accepted prophylactic doses instead, and three patients were inappropriately offered prophylactic regimes and were in effect undertreated. In the post-training group four patients with Wernicke's encephalopathy did not complete the treatment regime. With nursing staff persuasion they managed to receive between one and two doses/day and in theory were undertreated. Pain at injection site was the reason cited by all patients refusing the therapeutic regime.

We found that $15 \%(n=2)$ of the patients in the pre-training period who met the criteria for Wernicke's encephalopathy had ongoing monitoring of clinical signs to inform the treatment process, i.e. treatment discontinued if there was no clinical improvement. This increased in the post-training period to $68 \%$.

The ongoing monitoring of eye signs, confusion and ataxia following initiation of thiamine treatment regimes in the post-training sample provided an opportunity to record improvement on a daily basis. Results are charted in Fig. 1. The majority of the patients' eye signs and ataxia resolved within 2 days of initiation of treatment and all patients with ataxia recovered by the fifth day. Two patients continued to have nystagmus which did not respond to treatment at and
Table 3 Thiamine regimes administered and monitoring of clinical signs

\begin{tabular}{llc} 
& $\begin{array}{c}\text { Pre-training, } \\
N=40 \\
n(\%)\end{array}$ & $\begin{array}{c}\text { Post-training, } \\
N=55 \\
n(\%)\end{array}$ \\
\hline $\begin{array}{l}\text { Appropriate use of prophylactic } \\
\text { regime }\end{array}$ & $21(78)$ & $29(97)$ \\
\hline $\begin{array}{l}\text { Appropriate use of therapeutic } \\
\text { regime }\end{array}$ & $6(47)$ & $21(84)$ \\
\hline $\begin{array}{l}\text { Ongoing monitoring of clinical } \\
\text { signs among those started on } \\
\text { therapeutic regime }\end{array}$ & $2(15)$ & $20(68)$ \\
\hline
\end{tabular}

beyond day five and treatment was discontinued as per guidelines. Those presenting with acute confusion at admission $(n=3)$ recovered by the third day on the treatment regime.

\section{Discussion}

Nutritional deficiency of thiamine can lead to Wernicke's encephalopathy, and is most commonly seen in people with alcohol misuse. The condition is underrecognised and consequently undertreated, with disastrous implications for the patient and society as a whole. One factor contributing to the underrecognition could be poor awareness among doctors about current guidelines which emphasise a low threshold for clinical diagnosis and adequate treatment in the form of parenteral thiamine. Our results indicate that awareness of clinical presentation of Wernicke's encephalopathy among trainees is deficient and little has changed since Hope et $a l^{8}$ reported findings from their survey of A\&E physicians and psychiatrists. Further, knowledge of the appropriate treatment regimes for Wernicke's encephalopathy was also lacking.

This reflects in our baseline audit results, where only $46 \%$ of patients with presumptive diagnosis of Wernicke's encephalopathy were adequately treated, despite the availability of an effective and safe training. Following the introduction of education and training along with regular

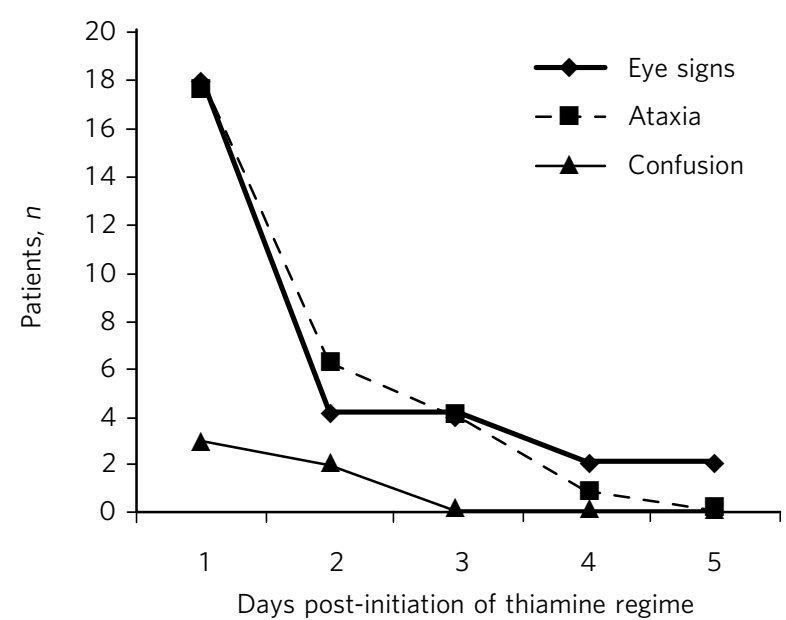

Fig 1 Resolution of eye signs (nystagmus/ophthalmoplegia), confusion and ataxia following initiation of thiamine regimes in the post-training sample. 
supervision, there was an increase in the proportion of patients being adequately treated in the re-audit. It is possible that the proportion of patients with Wernicke's encephalopathy that were recognised also improved across the audit period (33\% pre-training and $45 \%$ post-training). However, this could be reflective of true prevalence of presumptive Wernicke's encephalopathy in the differing samples.

The prevalence of presumptive Wernicke's encephalopathy overall across both sample populations was high (40\%) and is comparable to previous estimates among individuals with chronic alcohol misuse. It is also reflective of the audit setting, as alcohol detoxification units are tier 4 services in the current tiered approach adopted by community alcohol services. Also, individuals who are admitted for in-patient detoxification are those who are screened by the consultant addiction psychiatrist and considered high risk due to medical, psychiatric or social factors. Nonetheless, we feel that our findings are generalisable to detoxification units across the UK. They indicate that eye signs and ataxia are the most common signs of Wernicke's encephalopathy at presentation and the classic triad was present in less than $1 \%$ of our sample, which is lower than previous reports. This emphasises the point on the need for a low threshold to treat for Wernicke's encephalopathy in patients with alcohol misuse or dependence.

The lack of evidence-based treatment regimes to treat Wernicke's encephalopathy adds to the complexity around effective clinical management. For example, the Maudsley guidelines suggest discontinuation of therapeutic thiamine regime for presumptive diagnosis of Wernicke's encephalopathy if there is lack of clinical response after 2 days. However, others may view this as inadequate treatment and National Institute for Health and Care Excellence (NICE) guidelines published after completion of this audit recommend parenteral thiamine for a minimum of 5 days followed by oral supplementation. ${ }^{16}$

The results of the baseline audit revealed that ongoing monitoring of clinical signs for those with Wernicke's encephalopathy started on treatment regimes was deficient. This improved post-training and is important in view of completeness of treatment. The monitoring of these signs at re-audit was useful in establishing timescales of resolution of clinical signs in response to adequate treatment. Significant and rapid resolution of eye signs and confusion has been reported in a sample of 32 patients with acute Wernicke's encephalopathy after treatment with thiamine. ${ }^{17}$ Our findings mirror those described previously, in that eye signs including ophthalmoplegia and nystagmus resolved within the second day in a majority of patients with Wernicke's encephalopathy treated with the treatment regimes. Similarly, those presenting with acute confusion and ataxia responded favourably and rapidly to the regime.

\section{Trainee aspects}

Initial assessments are often carried out by trainees and it is therefore important that they are able to identify Wernicke's encephalopathy in order to initiate appropriate treatment, whether independently or under supervision. The clinical signs indicative of a presumptive diagnosis are largely bedside tests that are routinely carried out by trainees in similar units across the country.

The benefits of investing in current trainees could potentially bring longer-term rewards. Foundation year and GP trainees can translate this awareness into better standards of practice in other, non-psychiatric settings. Alcohol misuse and dependence are ubiquitous in psychiatric practice and therefore awareness of the condition and its treatment among psychiatry trainees is important. It could also be argued that as trainees are less established in their clinical practice they are more amenable to change.

The re-audit was not carried out with the same trainees as the baseline audit, which is a limitation. This was an unavoidable consequence of trainee rotation and the need to allow sufficient time before re-audit following training.

Last, the improvement in standards of practice could be attributable to the education and training, as well as the other component, namely regular supervision. It is our view that both components are equally important, as it has been repeatedly demonstrated that regular refresher sessions are a key factor in integrating interventions into routine clinical practice.

\section{About the authors}

Vijay Delaffon, Srinivas Naik and Rajandeep Mann are all specialty registrars in psychiatry, Leicestershire Partnership NHS Trust, Leicester, UK. Arshya Vahabzadeh is Resident in Psychiatry, Department of Psychiatry and Behavioral Health, Emory University, Atlanta, USA. Timothy Millward is a specialty registrar in psychiatry, Adeel Majeed and Angela Taylor are foundation trainees in psychiatry and Anshuman Pant is a consultant psychiatrist, all at Leicestershire Partnership NHS Trust, Leicester, UK.

\section{References}

1 Victor M, Adams RD, Collins GH. The Wernicke Korsakoff Syndrome and Related Neurological Disorders Due to Alcoholism and Malnutrition (2nd edn). FA Davis, 1989.

2 Cook CCH, Hallwood PM, Thomson AD. B Vitamin deficiency and neuropsychiatric syndromes in alcohol misuse. Alcohol Alcohol 1998; 33: 317-36.

3 Day E, Benthan P, Callaghan R, Kuruvilla T, George S. Thiamine for Wernicke-Korsakoff syndrome in people at risk from alcohol abuse. Cochrane Database Syst Rev 2004; 1: CD004003.

4 Torvik A. Wernicke's encephalopathy - prevalence and clinical spectrum. Alcohol Alcohol 1991; 26 (suppl 1): 381-4.

5 Harper C, Fornes P, Duyckaerts C, Lecomte D, Hauw J-J. An international perspective on the prevalence of the Wernicke-Korsakoff syndrome. Metab Brain Dis 1995; 10: 17-24.

6 Torvik A, Lindboe CF, Rogde S. Brain lesions in alcoholics: a neuropathological study with clinical correlations. J Neurol Sci 1982; 56: $233-48$

7 Blansjaar BA, Van Dijk JG. Korsakoff minus Wernicke syndrome. Alcohol Alcohol 1992; 27: 435-7.

8 Hope LC, Cook CCH, Thomson AD. A survey of the current clinical practice of psychiatrists and accident and emergency specialists in the UK concerning vitamin supplementation for chronic alcohol misusers. Alcohol Alcohol 1999; 34: 862-7.

9 Harper CG, Giles M, Finlay-Jones R. Clinical signs in the WernickeKorsakoff complex: a retrospective analysis of 131 cases diagnosed at necropsy. J Neurol Neurosurg Psychiatry 1986; 49: 341-5. 
10 Kopelman MD, Thomson AD, Guerrini I, Marshall EJ. The Korsakoff syndrome: clinical aspects, psychology and treatment. Alcohol Alcohol 2009; 44: 148-54.

11 Sullivan EV, Prefferbaum A. Neuroimaging of the Wernicke-Korsakoff syndrome. Alcohol Alcohol 2008; 118: 238-45

12 Mancinelli R, Ceccanti M. Biomarkers in alcohol misuse: their role in the prevention and detection of thiamine deficiency. Alcohol Alcohol 2009; 44: 177-82.

13 Thomson A, Cook C, Touquet R, Henry J. The Royal College of Physicians report on alcohol: guidelines for managing Wernicke's encephalopathy in the accident and emergency department. Alcohol Alcohol 2002; 37 513-21.
14 Day E, Callaghan R, Kuruvilla T, George S, Webb K, Bentham P. Pharmacybased intervention in Wernicke's encephalopathy. Psychiatrist 2010; 34 234-8.

15 Taylor D, Paton C, Kapur S. The South London and Maudsley NHS Foundation Trust and Oxleas NHS Foundation Trust Prescribing Guidelines (10th edn). Informa Healthcare, 2009.

16 National Institute for Health and Clinical Excellence. Alcohol Use Disorders: Diagnosis, Assessment and Management of Harmful Drinking and Alcohol Dependence (Clinical Guideline CG115). NICE, 2011.

17 Wood B, Currie J. Presentation of acute Wernicke's encephalopathy and treatment with thiamine. Metab Brain Dis 1995; 10: 57-72. 